\title{
Integrated guidance and control framework for the waypoint navigation of a miniature aircraft with highly coupled longitudinal and lateral dynamics
}

Proc IMechE Part G

$J$ Aerospace Engineering

$0(0) \mathrm{I}-13$

(C) IMechE 2020

Article reuse guidelines:

sagepub.com/journals-permissions DOI: 10.1 177/0954410020964992 journals.sagepub.com/home/pig

@SAGE

\author{
K Harikumar' ${ }^{1}$, Jinraj V Pushpangathan ${ }^{2}$, Sidhant Dhall ${ }^{2}$ and \\ M Seetharama Bhat $^{2}$
}

\begin{abstract}
A solution to the waypoint navigation problem for the fixed wing micro air vehicles (MAV) having a severe coupling between longitudinal and lateral dynamics, in the framework of integrated guidance and control (IGC) is addressed in this paper. IGC yields a single step solution to the waypoint navigation problem, unlike conventional multiple loop design. The pure proportional navigation (PPN) guidance law is integrated with the coupled MAV dynamics. A multivariable static output feedback (SOF) controller is designed for the linear state space model formulated in IGC framework. A waypoint navigation algorithm is proposed that handles the minimum turn radius constraint of the MAV and also evaluates the feasibility of reaching a waypoint. Non-linear simulations with and without wind disturbances are performed on a high fidelity $150 \mathrm{~mm}$ wingspan MAV model to demonstrate the proposed waypoint navigation algorithm.
\end{abstract}

\section{Keywords \\ Integrated guidance and control, coupled dynamics, micro air vehicle, static output feedback, waypoint navigation}

Date received: 14 November 2019; accepted: 9 September 2020

\section{Introduction}

Fixed wing micro air vehicles (MAV) are extremely agile, lightweight aircraft with a maximum wingspan of $150 \mathrm{~mm} .{ }^{1}$ In any of the MAV applications, it has to reach a set of waypoints, specified by the end user. In most of the waypoint navigation problems, guidance and control are treated as two separate problems. Control system is usually designed to give higher bandwidth to track the commands generated by the guidance law. But when the guidance law is integrated with the inner loop control system, the combined system often fails to meet the required performance and stability specifications. The major reasons are due to the assumption of lower order dynamics for the inner loop control system, neglecting actuator bandwidth and assuming decoupled longitudinal and lateral dynamics, especially for the case of MAVs. Many of the guidance law in the literature assumes lower order autopilot for inner loop control system. ${ }^{2}$ The assumption of lower order inner loop autopilot provides fictitious high amplitude gain margin for guidance law to operate. When the combined system is not stable, the guidance law parameters are retuned to ensure stability. The re-tuning of guidance law parameters often lead to inadequate performance. The actuator bandwidth is of major concern for MAVs. The short period mode and Dutch roll mode natural frequencies of MAVs are comparable to actuator bandwidth. ${ }^{3}$ The relatively lower actuator bandwidth in MAVs leads to poor wind disturbance rejection. The wind disturbances change angle of attack $(\alpha)$ and side slip angle $(\beta)$ considerably from the trim operating conditions. The additional forces and moments generated by wind disturbances are often neglected while designing the guidance law in the presence of wind. ${ }^{4}$ The coupling between longitudinal and lateral dynamics is severe for MAVs and cannot be neglected as in the case of bigger UAVs. ${ }^{3,5}$ The guidance law design needs to consider the

\footnotetext{
'International Institute of Information Technology Hyderabad, Hyderabad, India

${ }^{2}$ Indian Institute of Science, Bangalore, India

Corresponding author:

K Harikumar, International Institute of Information Technology Hyderabad, Hyderabad, India.

Email: harikumar.k@iiit.ac.in
} 
combined longitudinal and lateral dynamics, failure of which leads to instability during abrupt maneuvers.

The topic of integrated guidance and control (IGC) has gained considerable interest in the area of missile guidance and control ${ }^{6-11}$ due to the following advantages. The synergy between the guidance and control subsystems can be exploited to make the combined system more stable and effective than the individual subsystems. ${ }^{6}$ Despite the fact that separate guidance and control have been applied successfully, still there remains scope for further optimizing the performance and enhancing the stability. ${ }^{7}$ The prime focus on the missile IGC design is to achieve improved terminal miss distances. When the missile approaches the target, the time scale separation between the control loop and guidance loop is no longer valid. ${ }^{8}$ The conventional approach of designing separate guidance and control leads to a conservative design with the requirement of actuators with higher bandwidth than necessary. ${ }^{9}$

The idea of IGC is also applied to fixed wing UAVs. ${ }^{12-13}$ A linear time invariant model in the framework of integrated guidance and control is developed for a fixed wing UAV of wingspan $3.8 \mathrm{~m}$ to track helical trim trajectories in literature. ${ }^{12}$ For a UAV flying along the trim trajectory, the net forces and moments acting on the UAV are zero. The method developed in literature ${ }^{12}$ explains how to follow a given helical trajectory in which UAV remains in a trimmed state. But finding such a trimmed trajectory connecting two waypoints is very computationally intensive. Moreover, the method cannot be applied for in-flight waypoint changes since it is difficult to compute the trim trajectories online with the limited onboard computational power available with MAV. The IGC design for a fixed wing UAV of $8.8 \mathrm{~m}$ wingspan for following a predefined path using sliding mode control is explained in literature. ${ }^{14}$ The design assumes that the roll, pitch and yaw dynamics are decoupled. But for MAVs, the roll, pitch and yaw dynamics are tightly coupled. ${ }^{5}$ Nonlinear dynamic inversion (NDI) based IGC framework is developed for UAVs in literature $^{15-17}$ NDI utilizes a full state feedback structure. A combination of NDI and back-stepping method is used in literature ${ }^{18}$ for the IGC of a hypersonic vehicle. In the case of MAVs, measurement of $\alpha$ and $\beta$ is tedious due to the lack of availability of lightweight sensors. Moreover, the perturbations in $\alpha$ and $\beta$ are very high for the MAVs when compared to bigger UAVs under nominal wind conditions. IGC framework developed for lateral dynamics of a fixed wing UAV assumes decoupled longitudinal dynamics in literature. ${ }^{13}$ But the assumptions of decoupled longitudinal and lateral dynamics is not valid for $150 \mathrm{~mm}$ class of MAVs. ${ }^{3,5}$

To overcome the above-mentioned limitations of existing IGC methodology, a novel IGC framework combining the idea of pure proportional navigation
(PPN) with MAV dynamics is derived in this paper. The proposed waypoint navigation algorithm based on IGC framework is non-iterative and hence computationally inexpensive. The issue of coupling between longitudinal and lateral dynamics of the MAV is handled by the proposed IGC framework. Unlike the existing algorithms based on IGC framework, the proposed waypoint navigation algorithm evaluates the feasibility of reaching a waypoint based on the acceleration constraints of the MAV. The proposed IGC framework utilizes only measured outputs and does not require full state information as in literature. ${ }^{15-18} \mathrm{~A} H_{\infty}$ SOF controller is designed for the combined guidance and control loop using Linear Matrix Inequality (LMI) techniques. Numerical simulation results are presented for the straight line following and rectangular path following using a high fidelity nonlinear model of a $0.15 \mathrm{~m}$ wingspan MAV obtained from wind tunnel data. The simulations also show the wind disturbance attenuation capabilities of the IGC framework.

The paper is organized as follows. The coupled linear state space model of the MAV is presented in section Preliminaries. The guidance law used in IGC framework is given in section Guidance logic for waypoint navigation in two dimensional space. The integration of guidance law to the coupled dynamics of MAV and the waypoint navigation algorithm is presented in section Integrated guidance and control for waypoint navigation in two dimensional space. The non-linear six degrees of freedom simulations for straight line following and rectangular path following is given in section Numerical simulation results for waypoint navigation, followed by Conclusions.

\section{Preliminaries}

\section{Coupled linear state space model of the MAV}

The specifications of the MAV considered in this paper is given in Table 1. ${ }^{1,5}$ The MAV has a rectangular planform with a wingspan of $0.15 \mathrm{~m}$ and a chord length of $0.11 \mathrm{~m}$. Due to limitations in takeoff weight and space availability, only elevator and rudder control surfaces are employed without aileron. This makes the MAV further under-actuated when compared to conventional unmanned air vehicles (UAVs).

The coupling between longitudinal and lateral dynamics is severe for the case of MAVs. The coupled linear state space shows an unstable spiral mode, whereas the decoupled model fails to capture the unstable spiral mode. ${ }^{5}$ The effects of asymmetric propeller wake, motor-propeller counter torque and gyroscopic effects lead to unstable spiral mode dynamics. $^{5,19}$ The coupled linear state space model of the MAV is given below. ${ }^{5}$

$$
\dot{X}=A_{c} X+B_{c} U
$$


Table I. Specifications of the MAV.

\begin{tabular}{ll}
\hline Planform & Rectangular \\
\hline Take-off weight & 53 grams \\
Cruise airspeed & $8 \mathrm{~m} / \mathrm{s}$ \\
Stall airspeed & $6 \mathrm{~m} / \mathrm{s}$ \\
Wing - chord length & $0.11 \mathrm{~m}$ \\
Wing - span length & $0.15 \mathrm{~m}$ \\
Airfoil & E387 \\
Control surfaces & Elevator and rudder \\
\hline
\end{tabular}

where

$$
\begin{aligned}
& X=[\tilde{u}, \tilde{w}, \tilde{q}, \tilde{\theta}, \tilde{h}, \tilde{v}, \tilde{p}, \tilde{r}, \tilde{\phi}]^{T} \\
& \text { and } \\
& U=\left[\tilde{\delta}_{e s}, \tilde{\delta}_{r s}, \tilde{\delta}_{t}\right]^{T}
\end{aligned}
$$

The set $(\tilde{u}, \tilde{v}, \tilde{w})$ represents the linearized velocity along body axis, $(\tilde{p}, \tilde{q}, \tilde{r})$ are the linearized angular rates, $\tilde{\phi}$ is the linearized roll angle, $\tilde{\theta}$ is the linearized pitch angle and $\tilde{h}$ is the linearized altitude. The linearized elevator servo output $\tilde{\delta}_{e s}$, linearized rudder servo output $\tilde{\delta}_{r s}$ and the linearized thrust generated by motor-propeller denoted by $\tilde{\delta}_{t}$ are the control inputs. $A_{c}$ is the system matrix for coupled state space model and $B_{c}$ is the input matrix. The matrices $A_{c}$ and $B_{c}$ are given in ${ }^{5}$ for the case of $150 \mathrm{~mm}$ wingspan fixed wing MAV.

The states of longitudinal dynamics is given by

$$
X_{1}=[\tilde{u}, \tilde{w}, \tilde{q}, \tilde{\theta}, \tilde{h}]^{T}
$$

Similarly, the states of lateral dynamics is given by

$$
X_{2}=[\tilde{v}, \tilde{p}, \tilde{r}, \tilde{\phi}]^{T}
$$

The matrix $A_{c}$ in equation (1) can be written as

$$
A_{c}=\left(\begin{array}{ll}
A_{11} & A_{12} \\
A_{21} & A_{22}
\end{array}\right)
$$

where $A_{11}$ denotes the state matrix for decoupled longitudinal dynamics and $A_{22}$ the state matrix for decoupled lateral dynamics. The coupling between longitudinal and lateral dynamics is introduced by the matrices $A_{12}$ and $A_{21}$. For bigger UAVs, the eigenvalues of $A_{c}$ denoted by $\bar{\lambda}\left(A_{c}\right)=\bar{\lambda}\left(A_{11}\right) \cup$ $\bar{\lambda}\left(A_{22}\right)$. But for the case of $150 \mathrm{~mm}$ wingspan MAVs, $\bar{\lambda}\left(A_{c}\right) \neq \bar{\lambda}\left(A_{11}\right) \cup \bar{\lambda}\left(A_{22}\right){ }^{5}$

Remark: In the rest of this paper, a variable with symbol denotes the linearized variable. For example, the variables $(u, v, w)$ denotes the velocity components along the body axis and $(\tilde{u}, \tilde{v}, \tilde{w})$ represents their linearized version.

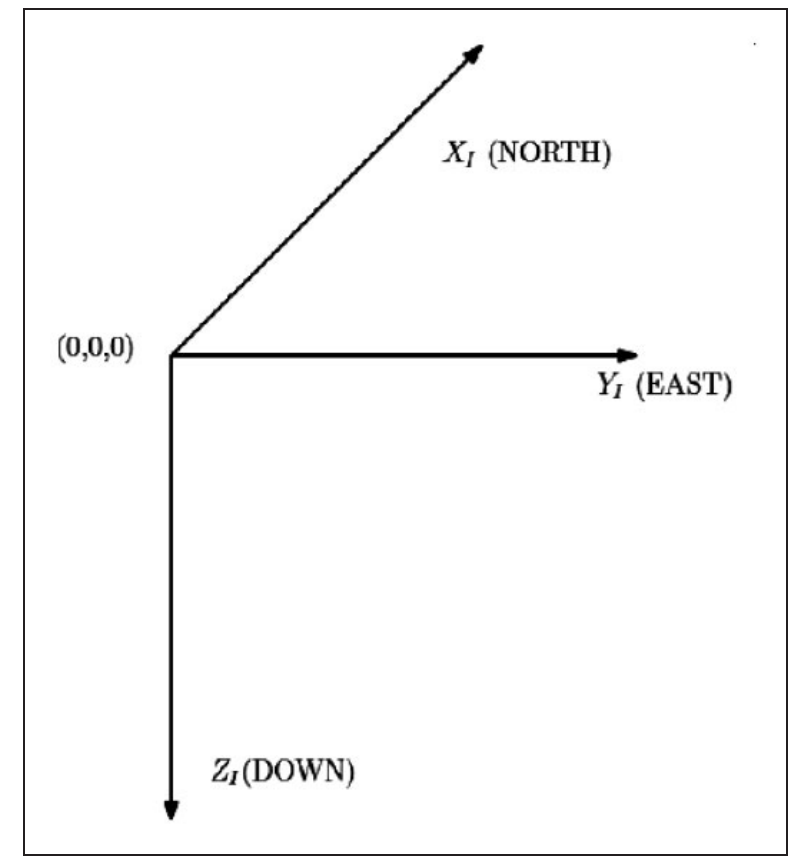

Figure I. Diagram showing inertial $X_{I} Y_{I} Z_{I}$ co-ordinate sytem.

Assumptions: The angle of sideslip $(\beta)$ is assumed to be negligible, the airspeed $\left(V_{a}\right)$ and altitude $(h)$ is assumed to be constant.

\section{Guidance logic for waypoint navigation in two dimensional space}

The origin of the local inertial coordinate system $\left(X_{I} Y_{I} Z_{I}\right)$ is the home location of the MAV as shown in Figure 1 with positive $X_{I}$ axis towards the geographic north pole, positive $Y_{I}$ axis in the direction of $90^{\circ}$ clockwise rotation from positive $X_{I}$ axis and positive $Z_{I}$ axis points towards the down direction. For the waypoint following in $2 \mathrm{D}$ space, altitude hold is employed using altimeter feedback, so motion in the local inertial $X_{I} Y_{I}$ plane is only considered. The guidance logic is explained first in this section and the integration of the guidance logic with the control system is explained later in section IV.

In Figure 2, axes $X^{\prime} Y^{\prime}$ are parallel to $X_{I} Y_{I},(x, y)$ is the current MAV position in the $X_{I} Y_{I}$ plane, $\left(x_{f}, y_{f}\right)$ is the next waypoint to be followed, $r_{a}$ is the range, $\sigma$ is the angle between the line joining $(x, y),\left(x_{f}, y_{f}\right)$ and the $Y^{\prime}$ axis, $\chi$ is the angle made by the velocity vector with the $X^{\prime}$ axis. The magnitude of the velocity vector is denoted by $V_{a}$. The miss distance $d$ is the perpendicular distance between $\left(x_{f}, y_{f}\right)$ and the velocity vector. The applied acceleration is denoted by $a_{c}$ and is perpendicular to the velocity vector and lies in the $X_{I} Y_{I}$ plane. This approach is suitable for fixed wing aircraft as the applied acceleration does not change the airspeed. ${ }^{20,21}$ The units of $(x, y)$, $\left(x_{f}, y_{f}\right), d, r_{a}$ are in meters $(\mathrm{m}), V_{a}$ is in meters/ second $(\mathrm{m} / \mathrm{s}), a_{c}$ is in meters/second-square $\left(\mathrm{m} / \mathrm{s}^{2}\right)$ and $\sigma, \chi$ are in radians(rad). From Figure 2, we can 


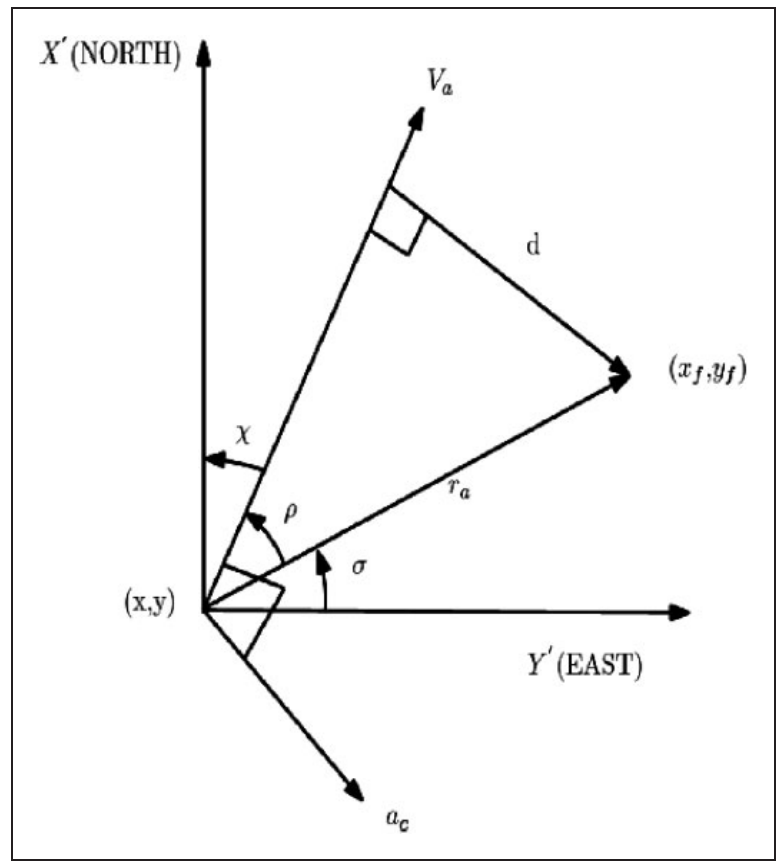

Figure 2. Diagram explaining the guidance law given in equation (I I).

write the following relations given in equations (7) to $(11)$.

The line-of-sight (LOS) angle $\sigma$ is given by

$$
\sigma=\tan ^{-1}\left(\frac{x_{f}-x}{y_{f}-y}\right)
$$

The angle $\rho$ is given by

$$
\rho=\frac{\pi}{2}-(\chi+\sigma)
$$

The range $r_{a}$ and miss distance $d$ are given in equations (9) and (10) respectively.

$$
\begin{aligned}
& r_{a}=\sqrt{\left(x_{f}-x\right)^{2}+\left(y_{f}-y\right)^{2}} \\
& d=r_{a} \sin \rho
\end{aligned}
$$

The applied acceleration $a_{c}$ is given by

$$
a_{c}=k_{1} d
$$

In equation (11), $k_{1}$ is the proportional gain. The magnitude of applied acceleration depends upon $k_{1}$. An expression for the gain $k_{1}$ is given in the next subsection. The acceleration is applied perpendicular to the velocity vector in such a way that it does not change the magnitude of velocity but only the direction. This is analogues to Pure Proportional Navigation (PPN) where the acceleration applied is perpendicular to the velocity vector. ${ }^{22}$ Here it is

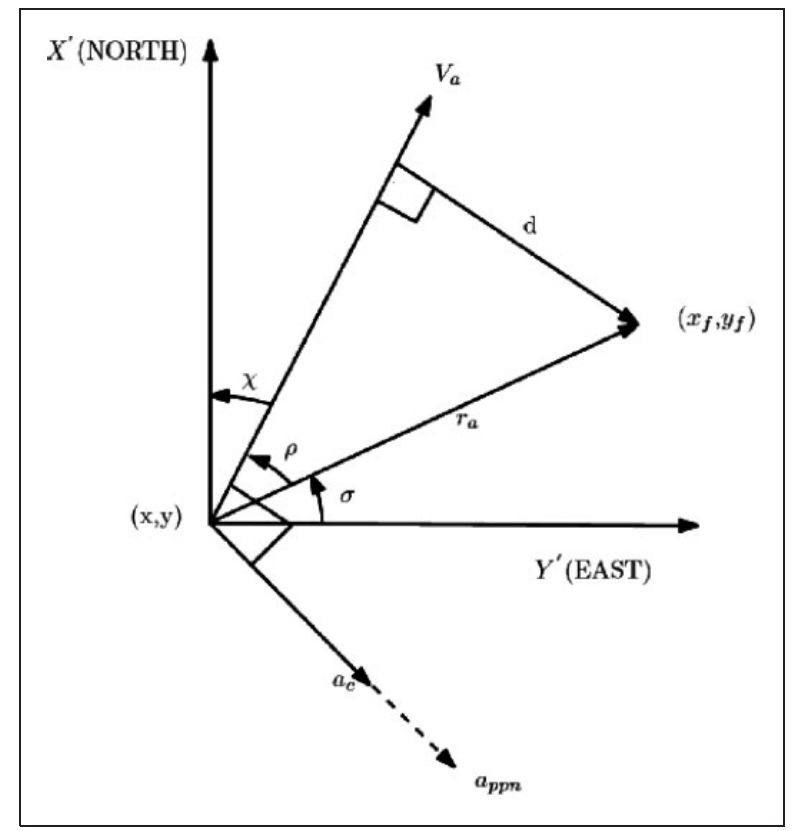

Figure 3. Diagram used for explaining the relation between the proposed guidance law and PPN guidance law.

assumed that altitude and $V_{a}$ is constant. When the miss distance is zero, from equation (10), we obtain either $r_{a}=0$ or $\rho=0$. The condition $r_{a}=0$ implies that $x=x_{f}$ and $y=y_{f}$. From Figure 2, $\rho=0$ implies that the velocity vector is pointing towards the goal point. So when the miss distance is zero, the MAV has either reached the desired waypoint or the velocity vector of the MAV is along the line connecting the waypoint and current MAV position such that no further acceleration has to be applied to change the direction of the MAV.

\section{Relation to PPN guidance law and choice of gain $k_{l}$}

This subsection gives the relation between the proposed guidance law given in equation (11) and the Pure Proportional Navigation (PPN) guidance law. Consider Figure 3, in which $a_{p p n}$ denotes the acceleration applied in PPN guidance law and rest all variables are same as in Figure 2. The expression for PPN guidance law ${ }^{22}$ is given by

$$
a_{p p n}=-N V_{a} \dot{\sigma}
$$

where $\dot{\sigma}$ is the line of sight rate and $N$ is the navigation constant.

$$
\dot{\sigma}=-\frac{V_{a} \sin \rho}{r_{a}}
$$

Using equations (12) to (13)

$a_{p p n}=N V_{a}\left(\frac{V_{a} \sin \rho}{r_{a}}\right)$ 
From equations (10) and (11)

$a_{c}=k_{1} r_{a} \sin \rho$

Substituting equations (15) into (14) gives

$$
a_{p p n}=\left(\frac{N}{k_{1}}\right)\left(\frac{V_{a}}{r_{a}}\right)^{2} a_{c}
$$

If we choose $k_{1}$ as given in equation (17), the proposed guidance law is same as the PPN guidance law. $^{23}$

$$
k_{1}=\frac{N\left(V_{a}\right)^{2}}{r_{a}^{2}}
$$

Thus the guidance law given in equation (11) takes the form

$$
a_{c}=\left(\frac{N\left(V_{a}\right)^{2}}{r_{a}^{2}}\right) d
$$

and is same as the PPN guidance law. The guidance law given in equation (18) will ensure that the MAV will reach the waypoint for $N>=2,{ }^{22}$

\section{Constraint on minimum turn radius}

Fixed wing MAVs have a constraint on the minimum allowable turn radius. The minimum value of the turn radius denoted by $R_{\min }$ depends upon the airspeed, stall angle of attack and the maximum thrust available. Since the applied acceleration is perpendicular to the velocity vector, the MAV follows a circular path with an instantaneous radius of curvature inversely proportional to the magnitude of the applied acceleration. The constraint on the applied acceleration $a_{c}$ can be given as in ${ }^{24}$

$$
a_{c}<\frac{V_{a}^{2}}{R_{\min }}
$$

Using equations (18) and (10), (19) can be written as

$$
\left(\frac{N\left(V_{a}\right)^{2}}{r_{a}^{2}}\right)\left(r_{a} \sin \rho\right)<\frac{V_{a}^{2}}{R_{\min }}
$$

After simplifying, equation (20) becomes

$$
\frac{N \sin \rho}{r_{a}}<\frac{1}{R_{\min }}
$$

The minimum value for $N=2$. Hence equation (21) becomes

$$
r_{a}>2 R_{\min } \sin \rho
$$

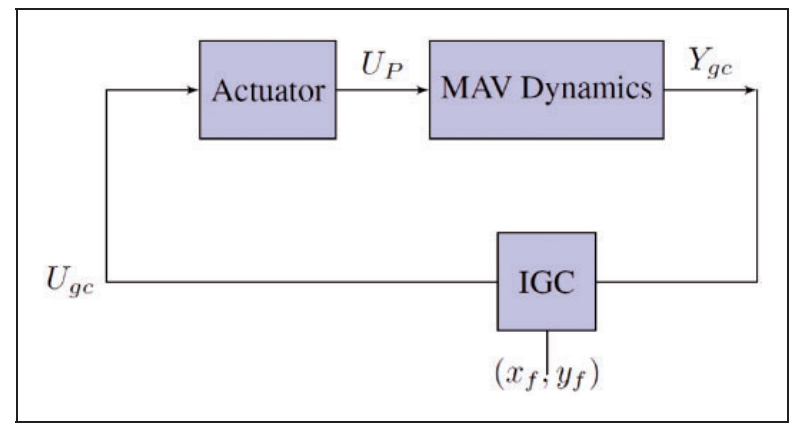

Figure 4. Architecture of integrated guidance and control for the MAV.

At any instant, the relation given by equation (22) ensures that the minimum turn radius constraint is not violated. A waypoint is considered to be not feasible if the condition given in equation (22) is violated.

\section{Integrated guidance and control for waypoint navigation in two dimensional space}

In separate guidance and control, the input to the actuator is computed in two sequential stages from the navigation data. Whereas in integrated guidance and control, the input to the actuator is computed in a single step from the navigation data. The block diagram of integrated guidance and control (IGC) is given in Figure 4. The integrated guidance and control block generates the actuator input $\left(U_{g c}\right)$ based on the output $\left(Y_{g c}\right)$ and the next waypoint position $\left(x_{n}, y_{n}\right) . U_{P}$ is the actuator output that deflects the elevator, rudder control surfaces and also changes the thrust input.

The measured output $Y_{I}$ is given by

$$
Y_{g c}=[q, \theta, h, p, r, \phi, d]^{T}
$$

The yaw angle $\psi$, the current position of the MAV $(x, y)$ and the next waypoint position $\left(x_{f}, y_{f}\right)$ is used for the computation of the miss distance $d$ as given in equations (7) to (10). Here the heading angle $\chi \approx \psi$ assuming the angle of side slip $(\beta)$ to be small.

Let $x_{1}=d$, and $x_{1}=\dot{d}=x_{2}$. The state variables used in the linear state space model of integrated guidance and control logic are

$$
X_{g c}=\left[X, X_{g}, X_{a}\right]^{T}
$$

where $X$ is given in equation (2)

$X_{g}=\left[\tilde{x}_{1}, \tilde{x}_{2}\right]$ are the states corresponding to the guidance logic and $X_{a}=\left[\tilde{\delta}_{e p}, \tilde{\delta}_{e p 1}, \tilde{\delta}_{e r}, \tilde{\delta}_{e r 1}\right]$ corresponds to second order elevator and rudder servo actuator transfer function. Thus

$$
X_{g c}=\left[\tilde{u}, \tilde{w}, \tilde{q}, \tilde{\theta}, \tilde{h}, \tilde{v}, \tilde{p}, \tilde{r}, \tilde{\phi}, \tilde{x}_{1}, \tilde{x}_{2}, \tilde{\delta}_{e p}, \tilde{\delta}_{e p 1}, \tilde{\delta}_{e r}, \tilde{\delta}_{e r 1}\right]^{T}
$$


In equation (25), $\tilde{x_{1}}, \tilde{x_{2}}$ are the variables obtained through the linearization of $x_{1}$ and $x_{2}$. The first step in the linearization process is to find a relation between $x_{2} \cdot(\ddot{d})$ and the acceleration command $a_{c}$. The second step is to find relation between $a_{c}$ and the turn rate of the vehicle which is a function of $\dot{\psi}$ and $\dot{\beta}$. These two steps will lead to the integration of $X_{g}=\left[\tilde{x}_{1}, \tilde{x}_{2}\right]$ to the state vector $X$ to form $X_{g c}$, since $\dot{\psi}$ and $\dot{\beta}$ is a function of $X$. As a first step, the commanded acceleration is simplified and is valid for small angles of $\rho$ as given in the below theorem.

Theorem I: For small angle $\rho$, the commanded acceleration can be written as

$$
a_{c}=\frac{1}{N-1} \ddot{d}
$$

Proof: The approximations $\sin \rho=\rho$ and $\cos \rho=1$ are done for small angle $\rho$. From equation (10)

$$
d=r_{a} \rho
$$

Differentiating the above equation gives the following

$$
\dot{d}=\dot{r}_{a} \rho+r_{a} \dot{\rho}
$$

From equation (9)

$$
\dot{r_{a}}=-V_{a}
$$

From equation (8)

$$
\dot{\rho}=-\dot{\chi}-\dot{\sigma}
$$
(18)

Using the relation $a_{c}=V_{a} \dot{\chi}$ and from equation

$$
\dot{\chi}=\frac{N V_{a} d}{r_{a}^{2}}
$$

From equations (13) and (10) we obtain

$$
\dot{\sigma}=-\frac{V_{a} d}{r_{a}^{2}}
$$

Substituting equations (31) and (32) into (30) gives

$$
\dot{\rho}=-(N-1) \frac{V_{a} d}{r_{a}^{2}}
$$

From equations (29), (33) and (28) we obtain

$$
\dot{d}=-\frac{N V_{a} d}{r_{a}}
$$

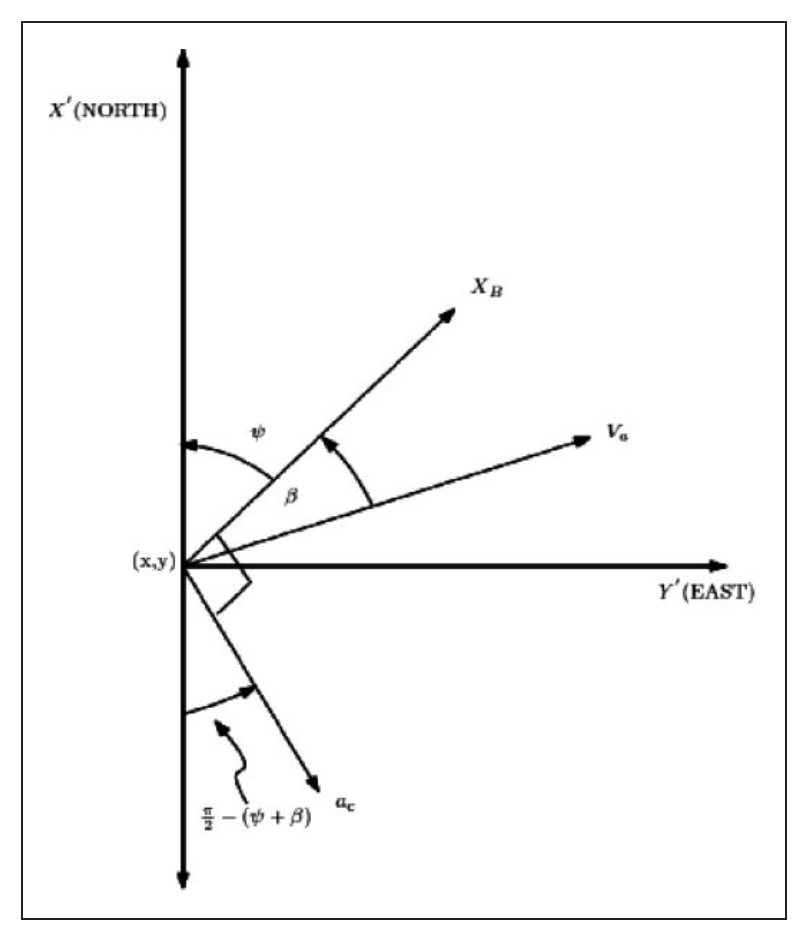

Figure 5. Body fixed lateral inertial plane of the MAV.

Differentiating the above equation and using equation (10) gives

$$
\ddot{d}=-N V_{a} \frac{d}{d t}(\rho)
$$

Using equations (31) and (32), (35) can be rewritten as

$$
\ddot{d}=(N-1) \frac{N V_{a}^{2} d}{r_{a}^{2}}
$$

Noting that $a_{c}=\frac{N V_{a}^{2} d}{r_{a}^{2}}$, the desired result is obtained.

The approximation of the non-linear guidance law that is valid for small angles of $\rho$ is given in equation (26). Now as the second step to enable the linearization process, a relationship between $a_{c}$ and $\psi, \beta$ is derived. In Figure 5, $X^{\prime} Y^{\prime}$ is the body fixed lateral inertial plane of the MAV with $X^{\prime}$ pointing towards north and $Y^{\prime}$ pointing towards east, $X_{B}$ denotes the body $\mathrm{X}$ axis, $\beta$ is the sideslip angle, $\psi$ is the yaw angle. The origin of the body fixed lateral inertial plane is the current position of the MAV denoted by $(x, y)$. When an acceleration $a_{c}$ is applied perpendicular to the velocity vector in the yaw plane as shown in the Figure 5, the MAV will move in a circular path in the body fixed lateral inertial plane with an angular velocity $\dot{\psi}+\dot{\beta}$. Then the applied acceleration can be equated to $V_{a}(\dot{\psi}+\dot{\beta})$ as given in equation (37). The heading angle $\chi=\psi+\beta$. From the Figure 5, we obtain

$$
a_{c}=V_{a}(\dot{\psi}+\dot{\beta})
$$


The expression for $\dot{\psi}$ and $\dot{\beta}$ is given in equations (38) and (39) respectively.

$$
\begin{aligned}
& \dot{\psi}=q \sin \phi \sec \theta+r \cos \phi \sec \theta \\
& \dot{\beta}=\frac{1}{V_{a}}\left(p w-r u+g \cos \theta \sin \phi+\frac{Y_{a}}{m}\right)
\end{aligned}
$$

where $Y_{a}$ is the side-force given by

$$
Y_{a}=\bar{Q} S C_{y}
$$

where $\bar{Q}=0.5 \rho_{a} V_{a}^{2}$ is the dynamic pressure in $\mathrm{N} / \mathrm{m}^{2}$ and $C_{y}$ is the side-force coefficient given by

$$
C_{y}=\left(C_{y \beta} \beta+C_{y p}\left(\frac{0.5 b}{V_{a}}\right) p+C_{y r}\left(\frac{0.5 b}{V_{a}}\right) r+C_{y \delta r} \delta_{r}\right)
$$

where $C_{y \beta}, C_{y p}, C_{y r}$ and $C_{y \delta_{r}}$ denotes the change in side-force coefficient due to $\beta, p, r$ and $\delta_{r}$ respectively.

From equations (37), (38) and (39) we can see that the applied acceleration $a_{c}$ is a function of the state variables and the rudder input.

Thus the state equations for the guidance logic are

$$
\begin{aligned}
& \dot{x_{1}}=x_{2} \\
& \dot{x_{2}}=(N-1) a_{c}
\end{aligned}
$$

Equations (42) and (43) are linearized and augmented with the MAV dynamic model to obtain the state space model for integrated guidance and control. Let the linearized variables for $x_{1}$ and $x_{2}$ be $\tilde{x}_{1}$ and $\tilde{x}_{2}$ respectively. Then the linear state equation and output equation for the integrated guidance and control for two dimensional space is given by

$$
\begin{aligned}
& \dot{X}_{g c}=A X_{g c}+B U_{g c} \\
& Y_{g c}=C X_{g c}
\end{aligned}
$$

where $X_{I}$ is given in equation (25) and

$$
\begin{aligned}
U_{g c} & =\left[\tilde{\delta}_{e}, \tilde{\delta}_{r}, \tilde{\delta}_{t}\right]^{T} \\
Y_{g c} & =\left[\tilde{q}, \tilde{\theta}, \tilde{h}, \tilde{p}, \tilde{r}, \tilde{\phi}, \tilde{x}_{1}\right]^{T}
\end{aligned}
$$

\section{Controller design for IGC framework}

For the trim conditions at $V_{a}=8 \mathrm{~m} / \mathrm{s}, \dot{\psi}=0.8 \mathrm{rad} / \mathrm{s}$ and climb rate $\dot{h}=1 \mathrm{~m} / \mathrm{s}$, the poles of linear state space model corresponding to IGC framework is given in Table 2. The spiral mode is unstable for the open loop system.

The controller is synthesized in $H_{\infty}$ robust control framework as it can handle uncertainties associated
Table 2. Poles of open loop IGC linear state space model.

\begin{tabular}{ll}
\hline Short period mode $\left(\omega_{s p}, \zeta_{s p}\right)$ & $35.6 \mathrm{rad} / \mathrm{s}, 0.285$ \\
Phugoid mode $\left(\omega_{p h}, \zeta_{p h}\right)$ & $1.15 \mathrm{rad} / \mathrm{s}, 0.219$ \\
Dutch roll mode, $\left(\omega_{d n} \zeta_{d r}\right)$ & $43.8 \mathrm{rad} / \mathrm{s}, 0.337$ \\
Roll subsidence mode & -2.44 \\
Spiral mode & 1.51 (unstable) \\
Elevator servo $\left(\omega_{e}, \zeta_{e}\right)$ & $48.7 \mathrm{rad} / \mathrm{s}, 0.742$ \\
Rudder servo $\left(\omega_{n}, \zeta_{r}\right)$ & $48.7 \mathrm{rad} / \mathrm{s}, 0.742$ \\
Poles for $\tilde{h}, \tilde{x_{1}}, \tilde{x_{2}}$ & $0,0,0$ \\
\hline
\end{tabular}

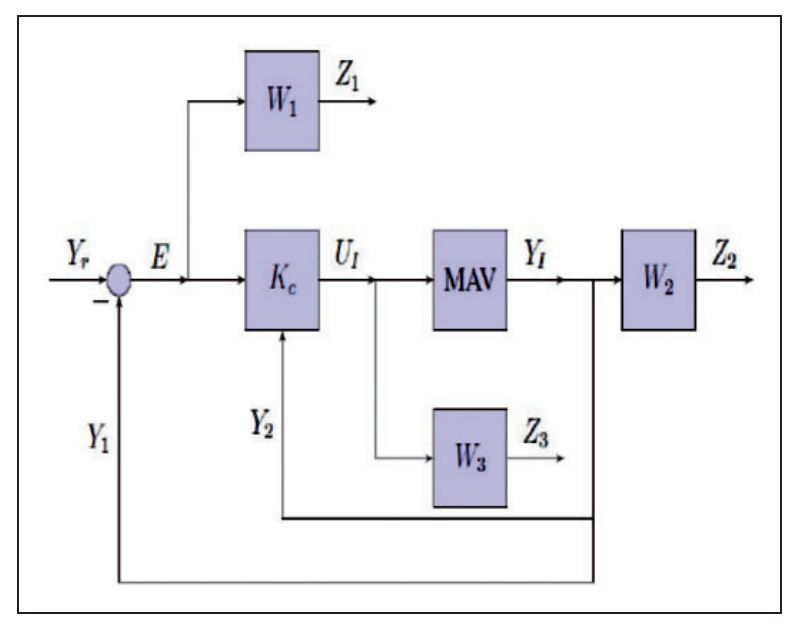

Figure 6. $H_{\infty}$ control architecture for IGC framework.

with the MAV dynamics. The architecture of $H_{\infty}$ control for IGC framework is shown in Figure 6. Here the reference signal $Y_{r}=\left[\tilde{h}_{r}, \tilde{x}_{1}\right]_{\tilde{\sigma}}^{T}$, and the measured outputs $Y_{1}=\left[\tilde{h}, \tilde{x_{1}}\right]^{T}, Y_{2}=[\tilde{q}, \tilde{\theta}, \tilde{p}, \tilde{r}, \tilde{\phi}]^{T}$. The weighting transfer functions $W_{1}, W_{2}$ and $W_{3}$ are low pass filter, high pass filter and constant respectively. ${ }^{25} Z_{1}, Z_{2}, Z_{3}$ are the performance outputs to be minimized.

The controller synthesis is performed in the discrete time domain using the algorithm described in. ${ }^{5}$ The controller sampling time is 0.02 seconds (the standard for servo control). The algorithm is summarized as given below. The discrete time equivalent linear state space model of the generalized plant shown in Figure 6 is given in equations (48) to (52).

$$
\begin{aligned}
& X_{d g}(k+1)=A_{d g} X_{d g}(k)+B_{d u} U_{d c}(k)+B_{d w} W_{d d}(k) \\
& Z_{d 1}(k)=C_{d 1} X_{d g}(k)+D_{d 11} U_{d c}(k)+D_{d 12} W_{d d}(k)
\end{aligned}
$$

$$
\begin{aligned}
& Z_{d 2}(k)=C_{d 2} X_{d g}(k)+D_{d 21} U_{d c}(k)+D_{d 22} W_{d d}(k) \\
& Z_{d 3}(k)=C_{d 3} X_{d g}(k)+D_{d 31} U_{d c}(k)+D_{d 32} W_{d d}(k)
\end{aligned}
$$

$$
Y_{d}(k)=C_{d} X_{d g}(k)
$$


The controller design objective is to synthesis a SOF control law

$$
U_{d c}(k)=F_{d} Y_{d}(k)
$$

that minimizes the weighted sensitivity $\left(Z_{1}=W_{1} S\right)$, weighted complimentary sensitivity $\left(Z_{2}=W_{2} T\right)$ and weighted control input sensitivity $\left(Z_{3}=W_{3} K S\right)$. The gain matrix $F_{d}$ is obtained by solving the LMI (linear matrix inequality) given in equation (54). The matrix $N_{d}$ is selected using the genetic algorithm (GA) for minimizing a performance index considering the closed loop damping ratio and stability of spiral mode and the resultant LMI is solved for $P_{d}$ and $F_{d}$. The matrix $P_{d}>0$ for a feasible solution $F_{d}$ that stabilizes the closed loop plant. The details of the control design is given in. ${ }^{5}$

$$
\left(\begin{array}{cc}
-P_{d} & -\left(A_{d g}+B_{d u} F_{d} C_{d}\right)^{T} N_{d}^{T} \\
-N_{d}\left(A_{d g}+B_{d u} F_{d} C_{d}\right) & P_{d}-N_{d}-N_{d}^{T}
\end{array}\right)<0
$$

The controller gain matrix $F_{d}$ is given in equation (55).where

$$
\begin{aligned}
& F_{d}=\left[F_{d 1}, F_{d 2}\right] \\
& F_{d 1}=\left[\begin{array}{ccc}
-0.0060 & -0.3378 & -0.8032 \\
0 & 0.0987 & 0 \\
-1.152 & 36.54 & 0.0042
\end{array}\right] \\
& F_{d 2}=\left[\begin{array}{cccc}
0.0002 & -0.0007 & 0 & -0.1008 \\
-0.0528 & 0.0008 & -0.0937 & -0.0002 \\
-5.325 & -19.1 & 0.0004 & 0.0091
\end{array}\right]
\end{aligned}
$$

For the trim conditions at $V_{a}=8 \mathrm{~m} / \mathrm{s}, \dot{\psi}=$ $0.8 \mathrm{rad} / \mathrm{s}$ and climb rate $\dot{h}=1 \mathrm{~m} / \mathrm{s}$, the dynamic modes of IGC closed loop linear state space model in continuous time domain is given in Table 3 for the navigation constant, $N=2$. From the Table 3 , we can see that the spiral mode is stabilized and the damping ratio of other modes have improved when compared to open loop plant. The three poles at the origin corresponding to $\tilde{h}, \tilde{x_{1}}$ and $\tilde{x_{2}}$ are placed in the left half of the s-plane.

A plot of sensitivity (S) and complementary sensitivity $(\mathrm{T})$ is given in Figure 7. From Figure 7, the closed loop system has good tracking and disturbance rejection performance till $4 \mathrm{rad} / \mathrm{s}$. A low value of $-20 \mathrm{~dB}$ is achieved for $\mathrm{T}$ beyond $10 \mathrm{rad} / \mathrm{s}$. This indicates rejection of sensor noise and robustness against unmodeled dynamics after a frequency of $10 \mathrm{rad} / \mathrm{s}$. A rise in the value of $\mathrm{T}$ between $30 \mathrm{rad} / \mathrm{s}$ and $40 \mathrm{rad} / \mathrm{s}$ is due to the closed loop natural frequency of short period and Dutch roll mode appearing in that range.
Table 3. Poles of closed loop IGC linear state space model.

\begin{tabular}{ll}
\hline Short period mode $\left(\omega_{s p}, \zeta_{s p}\right)$ & $36.8 \mathrm{rad} / \mathrm{s}, 0.404$ \\
Phugoid mode $\left(\omega_{p h}, \zeta_{p h}\right)$ & $2.25 \mathrm{rad} / \mathrm{s}, 0.323$ \\
Dutch roll mode, $\left(\omega_{d n} \zeta_{d r}\right)$ & $30.0 \mathrm{rad} / \mathrm{s}, 0.429$ \\
Roll subsidence mode & -15.5 \\
Spiral mode & -3.24 \\
Pole for $\tilde{h}$ & -1.50 \\
Poles for $\tilde{x_{l}}, \tilde{x_{2}}(\omega, \zeta)$ & $0.1 \mathrm{rad} / \mathrm{s}, 0.343$ \\
Elevator servo $\left(\omega_{\mathrm{e}}, \zeta_{\mathrm{e}}\right)$ & $44.4 \mathrm{rad} / \mathrm{s}, 0.671$ \\
Rudder servo $\left(\omega_{\mathrm{r}} \zeta_{r}\right)$ & $49.5 \mathrm{rad} / \mathrm{s}, 0.603$ \\
\hline
\end{tabular}

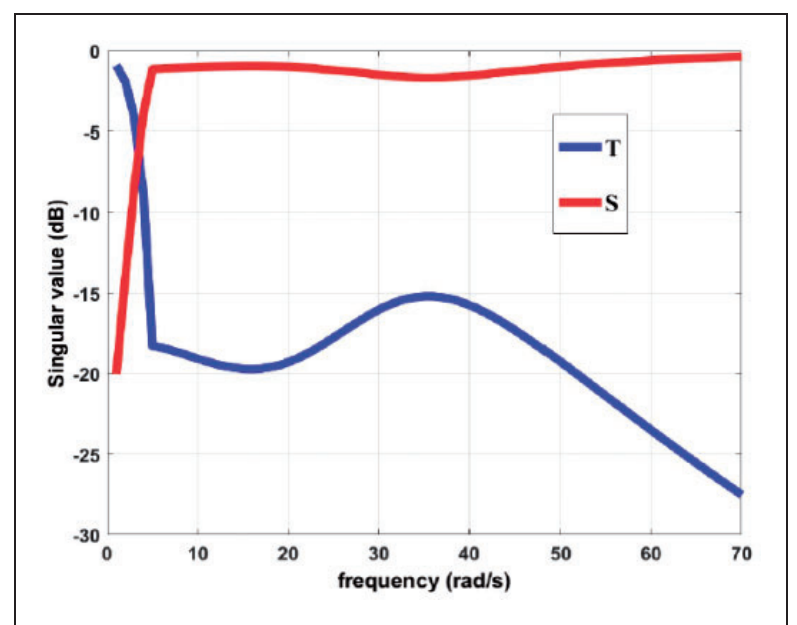

Figure 7. $\mathrm{S}$ and $\mathrm{T}$ plot for the closed loop IGC state space model.

\section{Waypoint navigation algorithm using IGC framework}

Inputs to the algorithm are the current $(x, y)$ position of the MAV obtained from GPS, the desired waypoint position $\left(x_{f}, y_{f}\right)$, altitude $h$ obtained from altimeter, rate gyro outputs p, q, r and Euler angles $\phi, \theta, \psi$. The steps are summarized in Algorithm I. For a given waypoint, the angle $\rho$ is computed from equation (8) and a constant roll angle command is issued if $|\rho|>20^{\circ}$. Here the small angle approximation $\sin \rho=$ $\rho$ and $\cos \rho=1$ is used for $|\rho| \leq 20^{\circ}$ and the expression given in equation (26) is valid for the same. The roll angle control for $150 \mathrm{~mm}$ MAV given in ${ }^{3}$ is used here. The altitude is maintained constant while executing the roll angle command. The MAV follows a constant roll angle command $\phi_{\text {ref }}=\operatorname{sgn}(\rho) \phi_{r}$ for $|\rho|>20^{\circ}$. Here $\phi_{r}$ is a constant value less than the maximum allowed roll angle $\phi_{\max }$ of the MAV. When $|\rho| \leq 20^{\circ}$, the feasibility of reaching the waypoint is verified using equation (19) and subsequently the control law given in equation (53) is employed as the IGC framework is valid. When the range $\left(r_{a}\right)$ is less than a pre-defined threshold $r_{t}$, the waypoint is considered to be reached and the entire process is repeated for a new waypoint. 


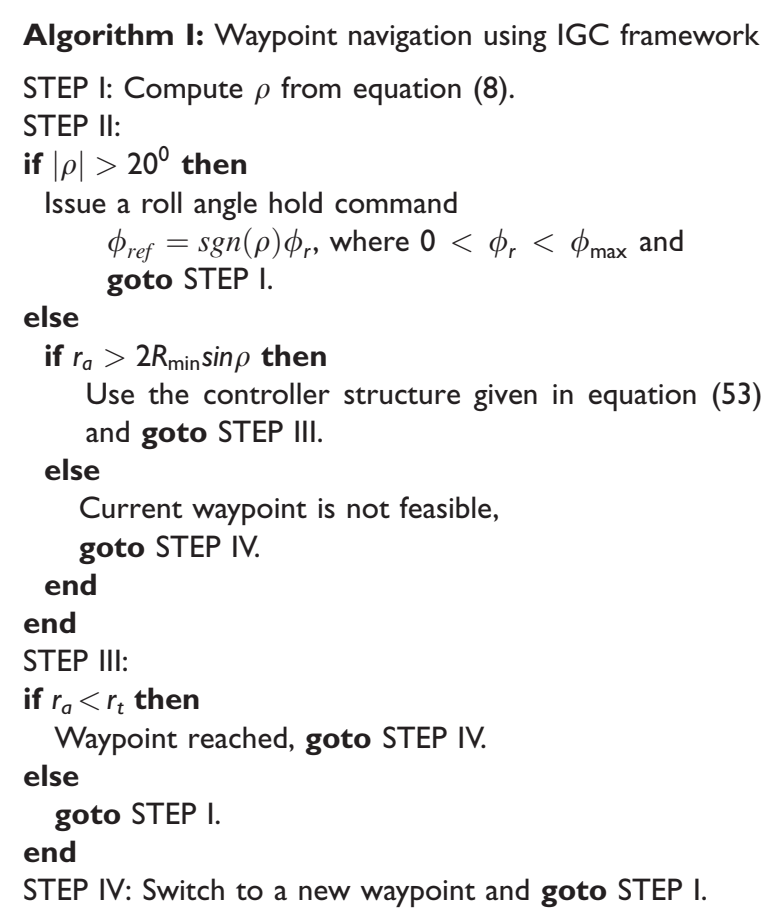

\section{Numerical simulation results for waypoint navigation}

The waypoint navigation algorithm is simulated for the non-linear six degrees of freedom (6DOF) model of the $150 \mathrm{~mm}$ MAV given in. ${ }^{1}$ Flight test results are presented in, ${ }^{1}$ validating the non-linear model used for simulation. Two simulations are performed in which the first one is to demonstrate straight line path following without any wind disturbances. The second simulation is for following a rectangular path without and with wind disturbances. Constraints are put on the actuators while performing the simulations. The range of elevator deflection is $\left[-35^{\circ}, 15^{\circ}\right]$, rudder deflection is $\left[-25^{\circ}, 25^{\circ}\right]$ and motor thrust is $0.0-0.45 \mathrm{~N}$. The second order transfer function model is used for elevator and rudder servos. The control input update is done at every 0.02 second. The $X Y$ position update is done at every 1 second. All other outputs are sampled at a rate of 0.02 second. The waypoint navigation logic is started when the MAV reach at an altitude of $20 \mathrm{~m}$ from the ground level. Altitude feedback is used to maintain the desired altitude of $20 \mathrm{~m}$. For both the simulations, the initial conditions are shown in Table 4.

\section{Straight line following}

The objective of the first simulation is to follow a straight line. The XY (North-East) path of the MAV is shown in Figure 8. In Figure 8, red circles represent the waypoints commanded and the solid blue line represents the path followed by the MAV. The plot of miss distance and the angle $\rho$ is given in Figure 9. During the initial stage, the miss distance is
Table 4. Initial conditions for the non-linear simulation.

\begin{tabular}{ll}
\hline Variable & Value \\
\hline$V_{a}, \alpha, \beta$ & $8 \mathrm{~m} / \mathrm{s}, 0.2286 \mathrm{rad},-0.0532 \mathrm{rad}$ \\
$\delta_{\mathrm{e}}, \delta_{r} \delta_{t}$ & $-0.2592 \mathrm{rad}, 0.1255 \mathrm{rad}, 0.23 \mathrm{I} \mathrm{N}$ \\
$\phi, \theta, \psi$ & $-0.036 \mathrm{I} \mathrm{rad}, 0.2304 \mathrm{rad}, 0 \mathrm{rad}$ \\
$\mathrm{p}, \mathrm{q}, \mathrm{r}$ & $0 \mathrm{rad} / \mathrm{s}, 0 \mathrm{rad} / \mathrm{s}, 0 \mathrm{rad} / \mathrm{s}$ \\
$\mathrm{u}, \mathrm{v}, \mathrm{w}$ & $7.78 \mathrm{~m} / \mathrm{s},-0.43 \mathrm{~m} / \mathrm{s}, 1.81 \mathrm{~m} / \mathrm{s}$ \\
$x, y, h$ & $0 \mathrm{~m}, 0 \mathrm{~m}, 20 \mathrm{~m}$ \\
\hline
\end{tabular}

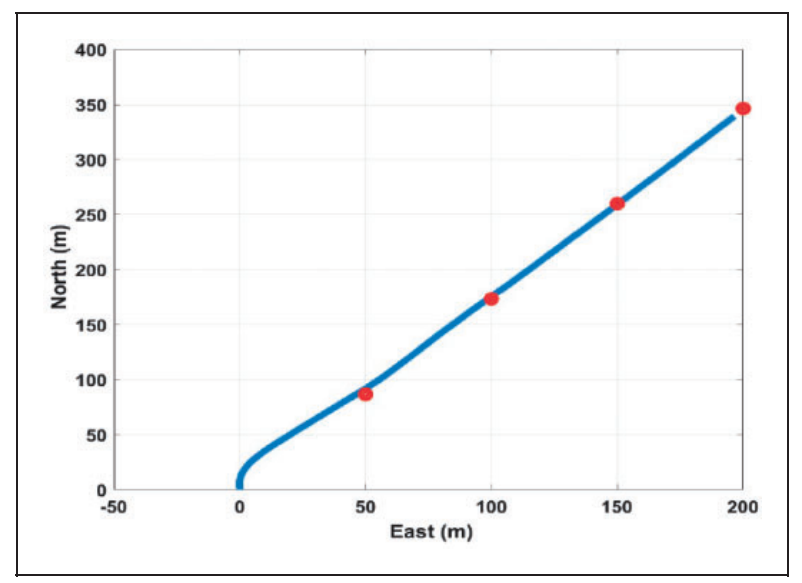

Figure 8. $X Y$ plot for MAV straight line following.

high as the first waypoint is not along the line-of-sight (LOS) of the MAV. After 25 seconds, we can see that the value of $\rho$ and miss distance is closer to zero as the MAV got aligned to the straight line. The small angle approximation used in the derivation of the IGC framework is used for $\rho<20^{\circ}$. Initially when $\rho>$ $20^{\circ}$ till 1.4 seconds, a constant roll angle command of $\phi_{r}=10^{\circ}$ is employed. A plot of Euler angles and angular rates are given in Figure 10. The roll angle $\phi$ goes to $10^{\circ}$ as shown in Figure 10 accordingly. The plot of control inputs is given in Figure 11. The peak value of elevator deflection is less than $-16^{\circ}$ and that of the rudder is less than $8^{\circ}$. The peak value of elevator deflection is less than $50 \%$ of the saturation value of $-35^{\circ}$. The peak value of rudder deflection is less than $33 \%$ of the saturation value of $25^{\circ}$. Thus the control inputs are well within the saturation limits. The variation airspeed is between $7.7 \mathrm{~m} / \mathrm{s}$ and $8.2 \mathrm{~m} /$ $s$ as seen from Figure 11 and is closer to the trim velocity of $8.0 \mathrm{~m} / \mathrm{s}$.

\section{Rectangular path following}

The objective of the second simulation is to follow a rectangular path with and without wind disturbances. A sinusoidal cross wind of amplitude $1 \mathrm{~m} / \mathrm{s}$ and frequency $0.5 \mathrm{rad} / \mathrm{s}$ is applied for the case with disturbances. Following a rectangular path enables the MAV to perform surveillance over a given area. 


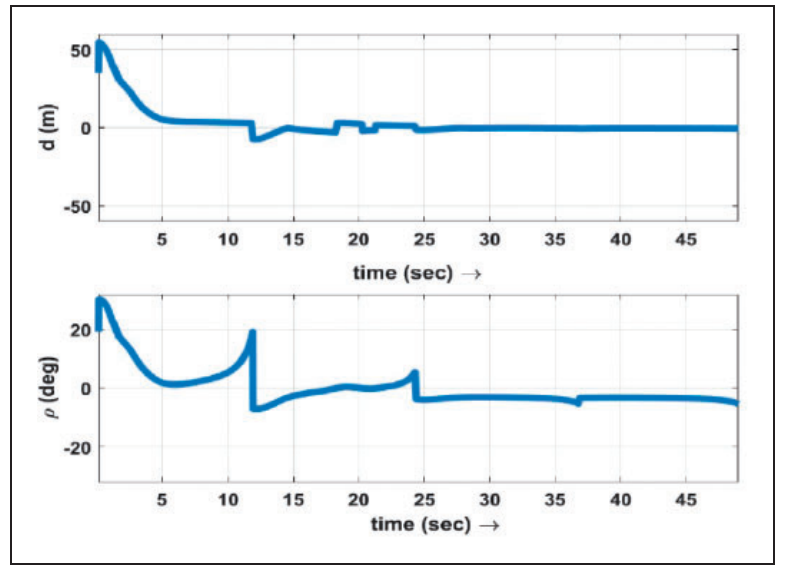

Figure 9. Miss distance and $\rho$ angle plots for MAV straight line following.

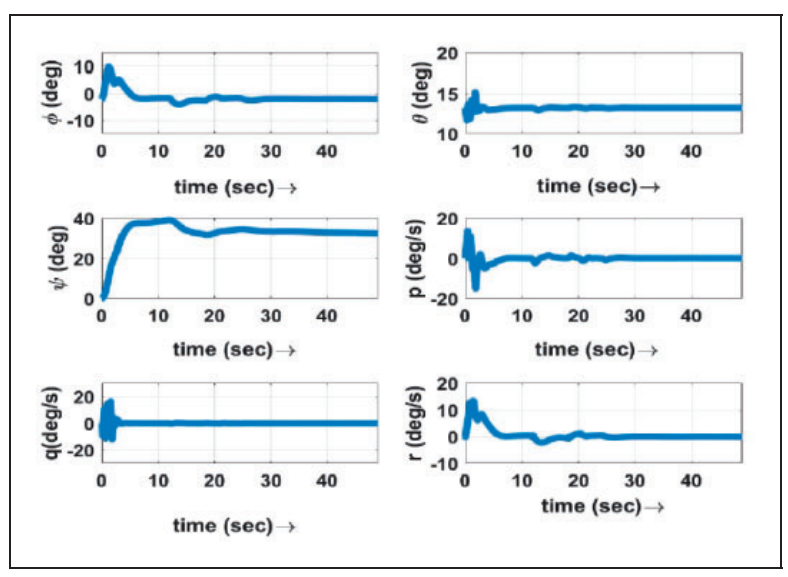

Figure 10. Euler angles and body axis angular rates for MAV straight line following.

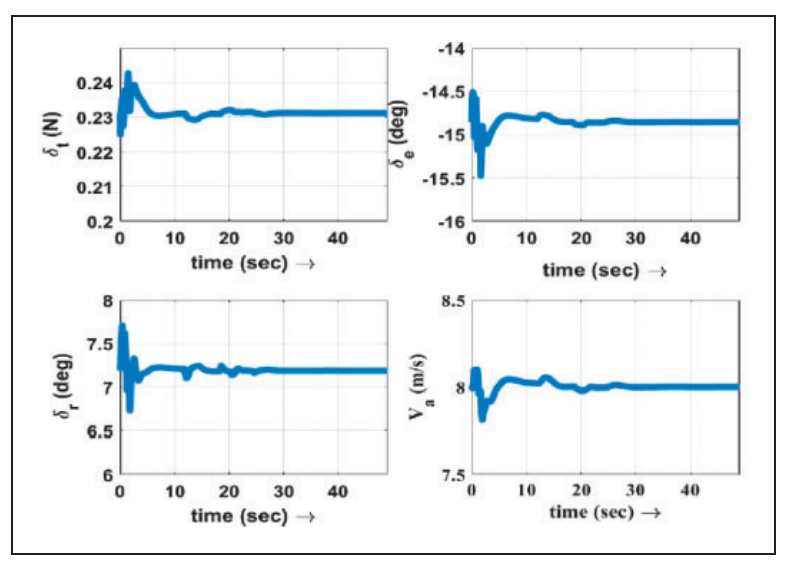

Figure II. Control inputs and airspeed for straight line following.

The rectangular path considered is of size $100 \mathrm{~m} \mathrm{x}$ $200 \mathrm{~m}$. The path followed by the MAV is shown in Figure 12 for the case without wind disturbances in solid blue lines and with wind disturbances in dotted black lines. The small red circles denotes the

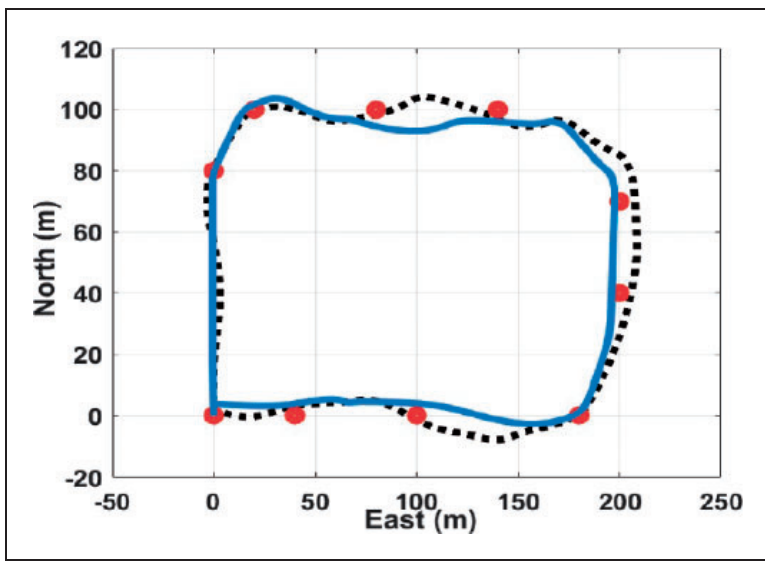

Figure 12. MAV rectangular path following: without wind (solid blue) and with wind (dotted black).

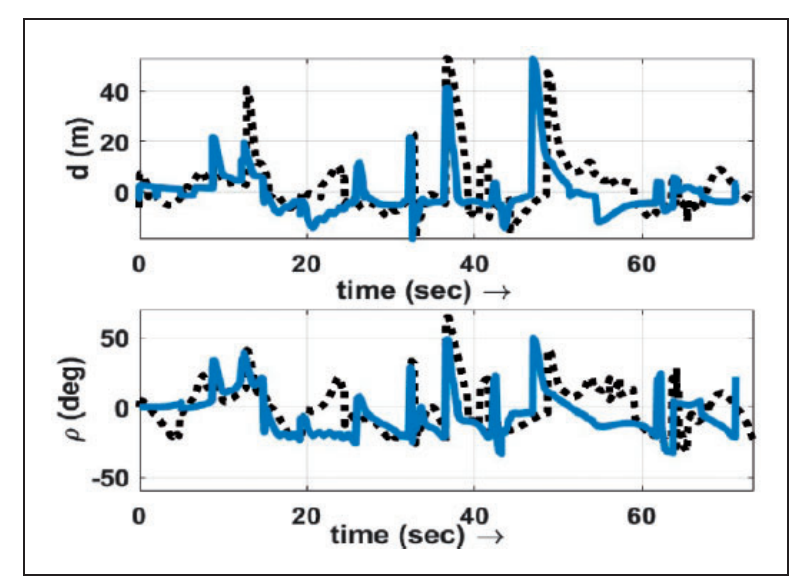

Figure 13. Miss distance and $\rho$ angle plots for rectangular path following: without wind (solid blue) and with wind (dotted black).

waypoints along the rectangular path. The starting point of the flight path is at $(0,0)$. For the case without wind disturbances, the MAV is able to follow a path closer to the desired path when compared to the path followed by the MAV with wind disturbances. The plot of $d$ and $\rho$ given in Figure 13 show oscillations whenever MAV switches to a new waypoint. The miss distance goes to zero as the MAV approaches a waypoint. The time taken to traverse all the waypoints is slightly higher for the case with wind disturbances when compared to no wind case. Similarly, the peak value of $d$ and $\rho$ is slightly higher for the case with wind disturbances. The simulation indicate that the closed loop IGC can compensate for wind disturbances of frequency less than $4 \mathrm{rad} / \mathrm{s}$ as given in Figure 7.

\section{Robustness against uncertainties}

To verify the robustness of the path following of MAV using IGC framework, five cases are considered with uncertainties in aerodynamic parameters, the 


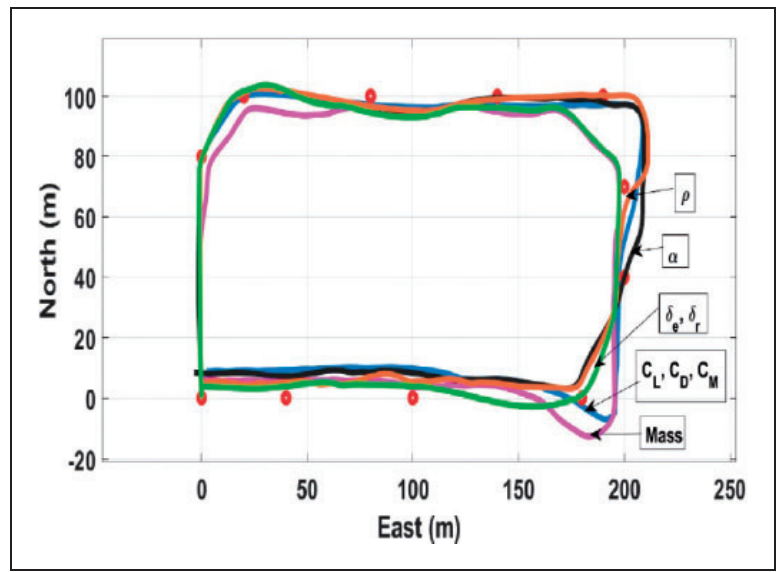

Figure 14. Path following using IGC under perturbations in different parameters.

mass of MAV, the initial condition of the angle of attack $(\alpha)$, initial conditions of control surfaces $\left(\delta_{e}\right.$, $\delta_{r}$ ) and air density $(\rho)$. The path followed by the MAV for the five cases is given in Figure 14. The aerodynamic parameters considered are the coefficient of lift $\left(C_{L}\right)$, coefficient of drag $\left(C_{D}\right)$ and the coefficient of pitching moment $\left(C_{M}\right)$. A simultaneous perturbation of $5 \%$ in $C_{L}, C_{D}$ and $10 \%$ in $C_{M}$ is applied (blue curve). Separate perturbations of $10 \%$ increase in the value of MAV mass (pink curve), $10 \%$ increase in the initial condition of $\alpha$ (black curve) from that given in Table 4 and $10 \%$ increase in the value of $\rho$ (orange curve) is applied respectively. Similarly, simulation corresponding to a simultaneous perturbation of $10 \%$ in initial conditions of $\delta_{e}$ and $\delta_{r}$ (green curve) from the value given in Table 4 is performed. The perturbations in mass result in maximum deviation from the desired path when compared to perturbations in other parameters. The increase in mass results in the reduction of the component of lift force available for turning manoeuver, and hence a larger turning radius. In all the five cases, the MAV is stable and hence the proposed IGC framework for path following is robust against parameter uncertainty, change in initial conditions and change in atmospheric conditions.

\section{Effect of navigation constant and sensor noise on the path following}

The magnitude of the commanded acceleration $a_{c}$ depends on the navigation constant $N$ for a fixed value of miss distance and range as given in equation (18). Simulations are performed for various values of $N \in\{2,3,4\}$ with IGC framework. The results are given in Figure 15 in the presence of measurement noise. For rate-gyro measurements, noise with a RMS value of $0.00105 \mathrm{rad} / \mathrm{s}$ is used corresponding to MPU9150 IMU (Inertial Measurement Unit). ${ }^{26}$ Noise with RMS value of $0.01 \mathrm{rad}$ is used for the Euler angle measurements $\phi, \theta, \psi$. The plot of miss

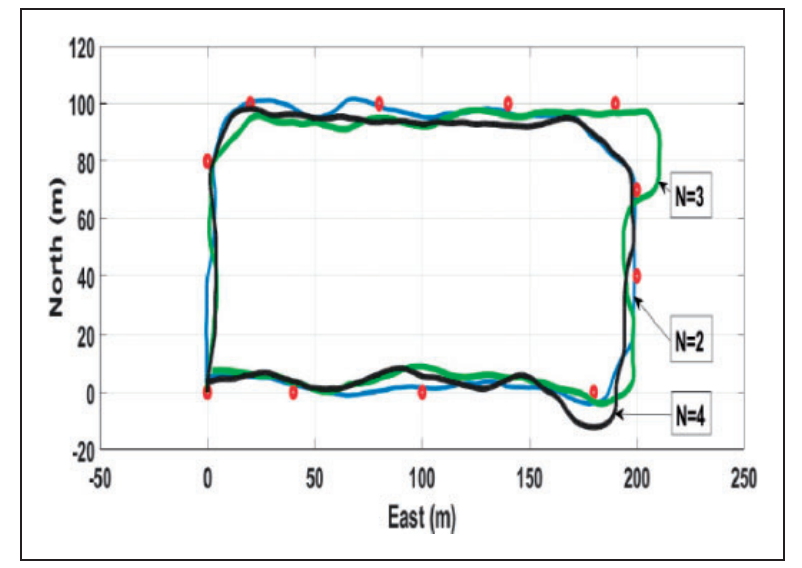

Figure 15. Path followed using IGC for different values of $N$ with sensor noise.

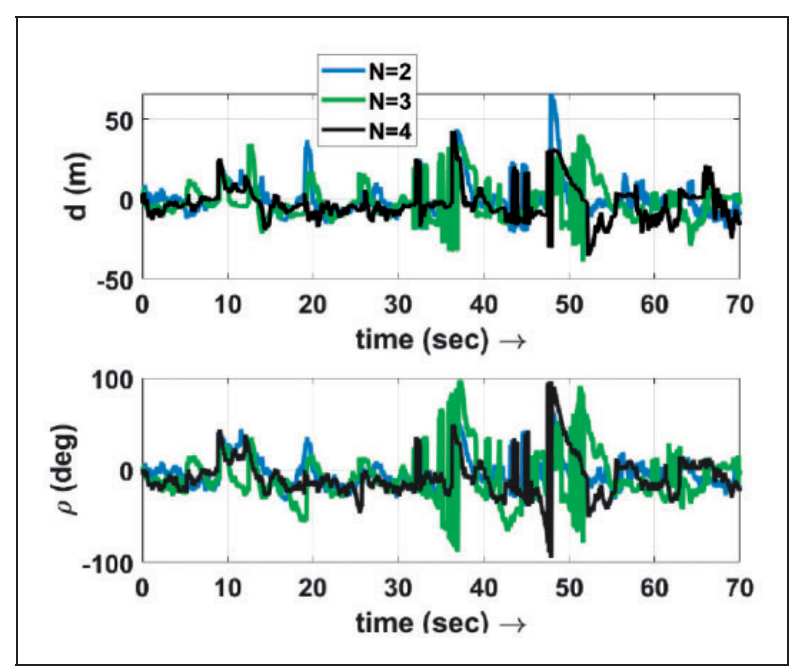

Figure 16. Miss distance and $\rho$ angle plots for path following using IGC for different values of $N$ with sensor noise.

distance and the angle $\rho$ is given in Figure 16. The deviation from the path is higher for $N=3$ when compared to $N=2,4$, as seen from Figure 15. This is because of the oscillations in $\rho$ for $N=3$, as seen in Figure 16. The lowest oscillations in $\rho$ are observed for $N=2$.

\section{Comparison with separate guidance and control}

A comparison between the proposed IGC framework based path following and the conventional separate guidance and control (SGC) is performed in this section. In separate guidance and control, the acceleration generated using equation (18) with $N=2$ is converted into equivalent roll angle command using the relation given below. ${ }^{27}$

$$
\phi_{\text {ref }}=\tan ^{-1}\left(\frac{a_{c}}{g}\right)
$$




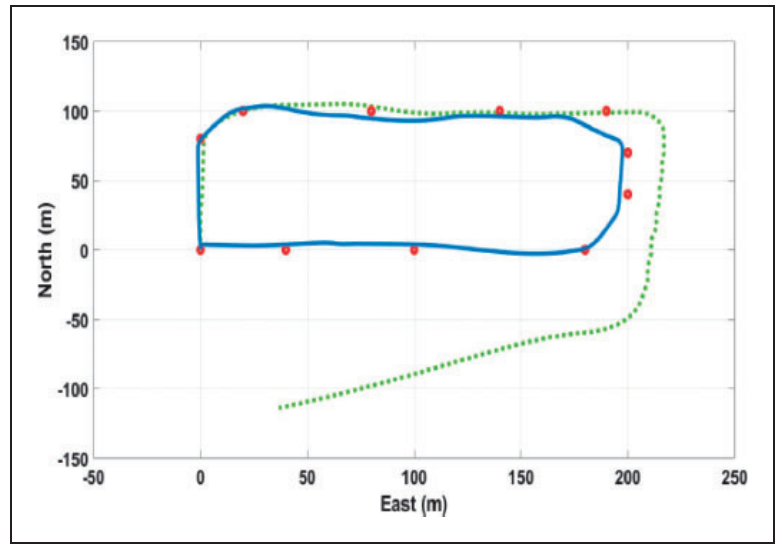

Figure 17. Path followed by IGC (solid blue) and SGC (dotted green) for $r_{t}=10 \mathrm{~m}$.

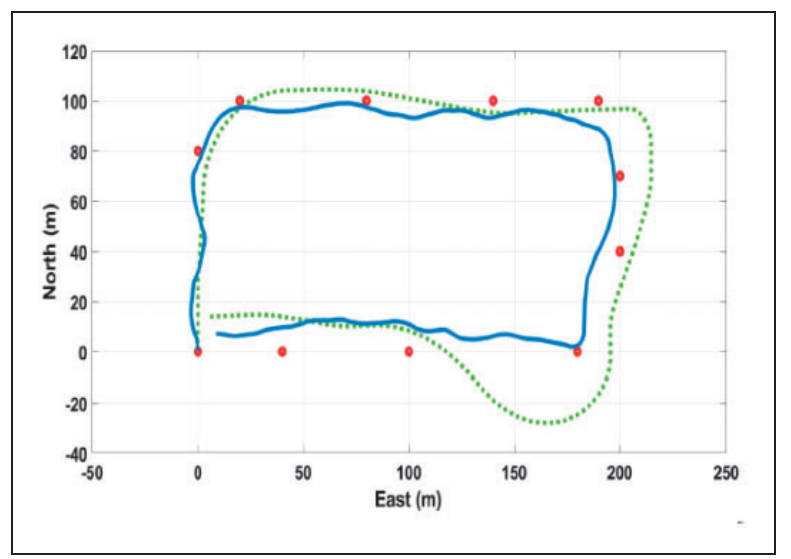

Figure 18. Path followed by IGC (solid blue) and SGC (dotted green) for $r_{t}=20 \mathrm{~m}$.

Simulation results are shown in Figure 17 for the case when the switching distance from one waypoint to another $r_{t}=10 \mathrm{~m}$. The MAV is able to follow the path using IGC (blue curve), while the SGC (dotted green curve) method diverges from the path after following the initial few waypoints. The switching distance, $r_{t}$, is increased to $20 \mathrm{~m}$ in the next simulation plot shown in Figure 18. The path followed using SGC is having a high deviation from the desired path when compared to the one followed using IGC. Thus the proposed IGC framework allows for tighter bounds on $r_{t}$ when compared to SGC, thereby improving the path following performance.

\section{Conclusions}

The paper presents a novel waypoint navigation algorithm in the IGC framework. The IGC methodology combines unstable MAV coupled dynamics with the pure proportional navigation guidance law. The resulting linear closed loop system is found to be stable with a static output feedback control law. The proposed waypoint navigation algorithm handles the minimum turn radius constraint of the MAV and determines the feasibility of reaching a given waypoint. The waypoint navigation algorithm does not require any iterative methods to compute the control inputs. The high fidelity nonlinear numerical simulations for straight line and rectangular path following validates the utility of the algorithm. The closed loop IGC framework is capable of attenuating the wind disturbances while path following. The airspeed of the MAV is maintained well above the stall value while performing the path following. The proposed IGC framework based path following is robust against parametric uncertainties and perturbations in initial flight conditions. The performance of the IGC framework based path following is shown to be better than path following based on separate guidance and control. The extension of this algorithm for waypoint navigation in three-dimensional space would be an interesting future work.

\section{Declaration of Conflicting Interests}

The author(s) declared no potential conflicts of interest with respect to the research, authorship, and/or publication of this article.

\section{Funding}

The author(s) disclosed receipt of the following financial support for the research, authorship, and/or publication of this article: The authors would like to thank National Programme for Micro Air Vehicle (NPMICAV) and Aeronautical Development and Research Board (ARDB), government of India for partial funding of the project.

\section{ORCID iD}

K Harikumar (D) https://orcid.org/0000-0002-5500-7133

\section{References}

1. Kandath H, Pushpangathan J, Bera T, et al. Modeling and closed loop flight testing of a fixed wing micro air vehicle. Micromachines 2018; 9: 111.

2. Nelson DR, Barber DB, McLain TW, et al. Vector field path following for miniature air vehicles. IEEE Trans Robot 2007; 23: 519-529.

3. Harikumar K, Dhall S and Bhat MS. Nonlinear modeling and control of coupled dynamics of a fixed wing micro air vehicle. In: 2016 Indian control conference (ICC), Hyderabad, India, 4 January 2016, pp. 318 323. Piscataway, New Jersey, USA: IEEE.

4. Hota S and Ghose D. Time-optimal convergence to a rectilinear path in the presence of wind. J Intell Robot Syst 2014; 74: 791-815.

5. Harikumar K, Dhall S and Bhat S. Design and experimental validation of a robust output feedback control for the coupled dynamics of a micro air vehicle. Int $J$ Control Autom Syst 2019; 17: 155-167.

6. Yan $\mathrm{H}$ and Ji H. Integrated guidance and control for dual-control missiles based on small-gain theorem. Automatica 2012; 48: 2686-2692. 
7. Xin M, Balakrishnan $\mathrm{SN}$ and Ohlmeyer EJ. Integrated guidance and control of missiles with $\theta$-D method. IEEE Trans Contr Syst Technol 2006; 14: 981-992.

8. Yi K, Tan Q, Liu S, et al. Integrated guidance and control design based on motion tracking. In: 2016 35th Chinese control conference (CCC) 2016. 27 Jul (pp. 5721-5725). IEEE.

9. Menon PK and Ohlmeyer EJ. Integrated design of agile missile guidance and autopilot systems. Control Eng Practice 2001; 9: 1095-1106.

10. Cong Z and Yun-Jie W. Non-singular terminal dynamic surface control based integrated guidance and control design and simulation. ISA Trans 2016; 63: 112-120.

11. Guo J, Xiong Y and Zhou J. A new sliding mode control design for integrated missile guidance and control system. Aerosp Sci Technol 2018; 78: 54-61.

12. Kaminer I, Pascoal A, Hallberg E, et al. Trajectory tracking for autonomous vehicles: an integrated approach to guidance and control. $J$ Guidance, Control, Dyn 1998; 21: 29-38.

13. Babar MZ, Samar R, Bhatti AI, et al. Robust integrated lateral guidance and control of UAVs. In: 2015 20th international conference on methods and models in automation and robotics (MMAR) 2015. 24 August (pp. 523-528). IEEE.

14. Yamasaki $\mathrm{T}$, Balakrishnan $\mathrm{SN}$ and Takano $\mathrm{H}$. Separate-channel integrated guidance and autopilot for automatic path-following. J Guidance, Control, Dyn 2013; 36: 25-34.

15. Padhi R, Rakesh PR and Venkataraman R. Formation flying with nonlinear partial integrated guidance and control. IEEE Trans Aerosp Electron Syst 2014; 50: 2847-2859.

16. Karimi $\mathbf{J}$ and Pourtakdoust SH. Integrated motion planning and trajectory control system for unmanned air vehicles. Proc IMechE, Part G: J Aerospace Engineering 2013; 227: 3-18.

17. Liu X, Huang W, Du L, et al. Three-dimensional integrated guidance and control for BTT aircraft constrained by terminal flight angles. In: The 27th Chinese control and decision conference (2015 CCDC) 2015. 23 May (pp. 107-112). IEEE.

18. Chao T, Zhang D, Wang $\mathrm{S}$, et al. Integrated guidance and control design considering system uncertainty. Proc IMechE, Part G: J Aerospace Engineering 2019; 233: 2278-2290.

19. Pushpangathan JV, Bhat MS and Harikumar K. Effects of gyroscopic coupling and countertorque in a fixedwing nano air vehicle. $J$ Aircraft 2018; 55: 239-250.

20. Roskam J. Airplane design part 6. Roskam Aviation Eng Corp 1990; 371-461.

21. Park S, Deyst J and How JP. Performance and lyapunov stability of a nonlinear path following guidance method. Journal of Guidance, Control, and Dynamics 2007; 30: 1718-1728.

22. Shneydor NA. Missile guidance and pursuit: kinematics, dynamics and control. Cambridge: Elsevier, 1998 pp.109-117.

23. Yanushevsky R. Guidance of unmanned aerial vehicles. USA: CRC Press, 2011, pp.9-18.

24. Meriam JL and Kraige LG. Engineering mechanics: dynamics. John Wiley \& Sons, 2008, pp.55-57.

25. $\mathrm{Hu}$ J, Bohn $\mathrm{C}$ and Wu HR. Systematic $H_{\infty}$ weighting function selection and its application to the real-time control of a vertical take-off aircraft. Control Eng Practice 2000; 8: 241-252.

26. InvenSense, MPU9150 IMU product specification, Document number: PS-MPU-9150A-00, Rev 4, 2012.

27. Stevens BL, Lewis FL and Johnson EN. Aircraft control and simulation: dynamics, controls design, and autonomous systems. John Wiley \& Sons, 2015. 